\title{
EXPERIMENTAL ANALYSIS OF MPTCP CONGESTION CONTROL ALGORITHMS; LIA, OLIA AND BALIA
}

\author{
Toshihiko Kato $^{1,2}$, Adhikari Diwakar ${ }^{1}$, Ryo Yamamoto $^{1}$, Satoshi Ohzahata ${ }^{1}$ \\ and Nobuo Suzuki ${ }^{2,3}$ \\ ${ }^{1}$ University of Electro-Communications \\ 1-5-1, Chofugaoka, Chofu, Tokyo 182-8585, Japan \\ ${ }^{2}$ Advanced Telecommunication Research Institute International \\ 2-2-2, Hikaridai, Seika-cho, Sorakugun, Kyoto 619-0288, Japan \\ ${ }^{3}$ Kindai University \\ 11-6, Kashiwa-no-mori, Iizuka, Fukuoka 820-0011, Japan
}

\begin{abstract}
In Multipath TCP (MPTCP), the congestion control is realized by individual subflows (conventional TCP connections). However, there is some concern that the total data sending rate becomes too high compared with other single path TCPs if the associated subflows increase their own congestion windows independently. So, a coupled increase scheme of congestion windows, called Linked Increase Adaptation (LIA), is adopted as a standard congestion control algorithm for subflows comprising a MPTCP connection. But this algorithm supposes that TCP connections use Additive Increase and Multiplicative Decrease (AIMD) based congestion control, and if high speed algorithms such as CUBIC TCP are used, the throughput of MPTCP connections might be decreased. In order to increase the throughput of MPTCP connection, two modified congestion control algorithms, Opportunistic Linked-Increase Algorithm (OLIA) and Balanced Linked Adaptation (BALIA) are also introduced. However, they are still based on AIMD and there is some concern that they are weaker than high speed algorithms. This paper analyzes the performance of MPTCP congestion control algorithms through experiments. Specifically, this paper examines to compare LIA, OLIA or BALIA based MPTCP flow and a single TCP flow with TCP Reno or CUBIC TCP. These experiments show that LIA is weaker than both Reno and CUBIC, and OLIA and BALIA are weaker than CUBIC.
\end{abstract}

\section{KEYWORDS}

MPTCP, Congestion Control, LIA, OLIA, BALIA, TCP Reno, CUBIC TCP

\section{INTRODUCTION}

Recent mobile terminals are equipped with multiple interfaces. For example, most smart phones have interfaces for 4G Long Term Evolution (LTE) and Wireless LAN (WLAN). In the next generation (5G) mobile network, it is expected that mobile terminals will be equipped with more interfaces by using multiple communication paths provided multiple network operators [NGNM Alliance, 2015]. However, the conventional Transmission Control Protocol (TCP) establishes a connection between a single IP address at either end, and so it cannot handle multiple interfaces at the same time. In order to utilize the multiple interface configuration, Multipath TCP (MPTCP) [Paasch and Bonaventure, 2014], which is an extension of TCP, has been introduced in several operating systems, such as Linux, Apple OS/iOS [Apple Insider Staff, 2019] and Android [icteam, 2019]. Conventional TCP applications can use MPTCP as if they were working over conventional TCP and are provided with multiple byte streams through different interfaces.

MPTCP is defined in three Request for Comments (RFC) documents by the Internet Engineering Task Force. RFC 6182 [Ford et al., 2011] outlines architecture guidelines. RFC 6824 [Ford et al., 2013] presents the details of extensions to support multipath operation, including the maintenance of an MPTCP connection and subflows (TCP connections associated with an MPTCP connection), and the data transfer over an MPTCP connection. RFC 6356 [Raiciu et al., 2011] presents a congestion control algorithm that couples the congestion control algorithms running on different subflows. 
One significant point on the MPTCP congestion control is that, even in MPTCP, individual subflows perform their own control. RFC 6356 requires that an MPTCP data stream do not provide too large throughput compared with other (single) TCP data streams sharing a congested link. For this purpose, RFC 6356 defines an algorithm called Linked Increase Adaptation (LIA), which couples and suppresses the congestion window size of individual subflows. Besides, more aggressive algorithms, such as Opportunistic Linked-Increases Algorithm (OLIA) [Kalili et al., 2013] and Balanced Linked Adaptation (BALIA) [Peng et al., 2016], are proposed. However, all of those algorithms are based on the Additive Increase and Multiplicative Decrease (AIMD) scheme like TCP Reno [Floyd et al., 2004]. That is, the increase of congestion window at receiving a new ACK segment is in the order of $1 /$ (congestion window size). On the other hand, current modern operating systems uses high speed congestion control algorithms, such as CUBIC TCP [Ha et al., 2008] and Compound TCP [Tan et al., 2006]. These algorithms increase the congestion window more aggressively than TCP Reno. So, it is possible that the throughput of LIA and other MPTCP congestion control algorithms is suppressed when it coexists with them.

Based on these considerations, we presented the results of performance evaluation in our previous paper [Kato et al., 2019]. Specifically, we conducted two kinds of experiments. One is to replace the congestion control algorithm of MPTCP from LIA to TCP Reno or CUBIC TCP. The other is for evaluating the performance when a MPTCP connection with LIA and a single path TCP with TCP Reno / CUBIC TCP share a bottleneck link. The results of two experiments showed that the throughput of LIA is lower than that of TCP Reno and CUBIC TCP. But, the experiments have some issues that the number of experimental runs are limited and that the MPTCP used Ethernet and WLAN interfaces, the latter of which may decrease the MPTCP throughput. So, this paper presents more detailed and fair performance experiment and also gives the results for OLIA and BALIA. The rest of this paper is organized as follows. Section II explains the overview of MPTCP and the details of MPTCP congestion control algorithms. Section III describes the experimental setting for performance evaluation. Section IV shows the results of the performance evaluation where LIA, OLIA and BALIA coexist together with TCP Reno and CUBIC TCP over a bottleneck link. In the end, Section V concludes this paper.

\section{BACKGROUNDS}

\subsection{Overview of MPTCP}

As described in Figure 1, the MPTCP module is located on top of TCP. MPTCP is designed so that the conventional applications do not need to care about the existence of MPTCP. MPTCP establishes an MPTCP connection associated with two or more regular TCP connections called subflows. The management and data transfer over an MPTCP connection is done by newly introduced TCP options for the MPTCP operations.

When the first subflow is established, a TCP option called MP_CAPABLE is used within SYN, SYN+ACK, and the following ACK segments. When the following subflows are established, the MP_JOIN option is used so that the new TCP connections are associated with the existing MPTCP connection.

An MPTCP implementation will take one input data stream from an application, and split it into one or more subflows, with sufficient control information to allow it to be reassembled and delivered to the receiver side application reliably and in order. An MPTCP connection maintains the data sequence number independent of the subflow level sequence numbers. The data and ACK segments may contain a Data Sequence Signal (DSS) option depicted in Figure 2.

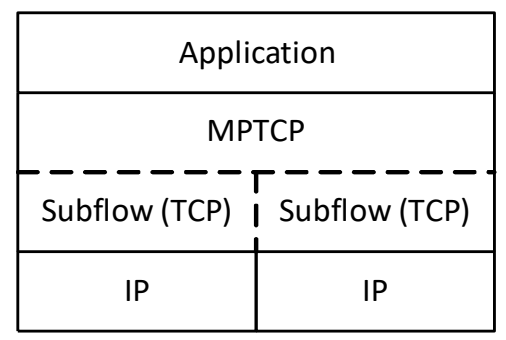

Figure 1. Layer structure of MPTCP

\begin{tabular}{|c|c|c|c|}
\hline Kind $(=30)$ & Length & $\begin{array}{c}\text { Subtype } \\
(=2)\end{array}$ & Flags \\
\hline \multicolumn{3}{|c|}{ Data ACK ( 4 or 8 bytes, depending on flags) } \\
\hline \multicolumn{2}{|c|}{ Data sequence number ( 4 or 8 bytes, depending on flags) } \\
\hline \multicolumn{2}{|c|}{ Subflow sequence number (4 bytes) } \\
\hline Data-level length ( 2 bytes) & Checksum ( 2 bytes) \\
\hline
\end{tabular}

Figure 2. Data Sequence Signal option 
The data sequence number and data ACK is 4 or 8 byte long, depending on the flags in the option. The number is assigned on a byte-by-byte basis similarly with the TCP sequence number. The value of data sequence number is the number assigned to the first byte conveyed in that TCP segment. The data sequence number, subflow sequence number (relative value) and data-level length define the mapping between the MPTCP connection level and the subflow level. The data ACK is analogous to the behavior of the standard TCP cumulative ACK. It specifies the next data sequence number a receiver expects to receive.

\subsection{Overview of MPTCP Congestion Control}

\subsubsection{LIA}

As described above, in MPTCP, only subflows manage their congestion windows, that is, an MPTCP connection does not have its congestion window size. Under this condition, if subflows perform their congestion control independently, the throughput of MPTCP connection will be larger than single TCP connections sharing a bottleneck link. RFC 6356 decides that such a method is unfair for conventional TCP. RFC 6356 introduces the following three requirements for the congestion control for MPTCP connection.

- Goal 1 (Improve throughput): An MPTCP flow should perform at least as well as a single TCP flow would on the best of the paths available to it.

- Goal 2 (Do no harm): All MPTCP subflows on one link should not take more capacity than a single TCP flow would get on this link.

- Goal 3 (Balance congestion): An MPTCP connection should use individual subflow dependent on the congestion on the path.

In order to satisfy these three goals, RC6356 proposes an algorithm that couples the additive increase function of the subflows, and uses unmodified decreasing behavior in case of a packet loss. This algorithm is called LIA and summarized in the following way.

Let $c w n d_{i}$ and $c w n d_{-}$total be the congestion window size on subflow $i$, and the sum of the congestion window sizes of all subflows in an MPTCP connection, respectively. Here, we assume they are maintained in packets. Let $r t t_{i}$ be the Round-Trip Time (RTT) on subflow $i$. For each ACK received on subflow $i, c w n d_{i}$ is increased by

$$
\min \left(\frac{\alpha}{\text { cwnd_total }}, \frac{1}{\text { cwnd }}\right) \text {. }
$$

The first argument of min function is designed to satisfy Goal 2 requirement. Here, $\alpha$ is defined by

$$
\alpha=\text { cwnd_total } \cdot \frac{\max _{k}\left(\frac{c w n d_{k}}{r t t_{k}}\right)}{\left(\sum_{k} \frac{c w n d_{k}}{r t t_{k}}\right)^{2}} .
$$

By substituting (2) to (1), we obtain the following equation.

$$
\min \left(\frac{\max _{k}\left(\frac{c w n d_{k}}{r t t_{k}^{2}}\right)}{\left(\sum_{k} \frac{c w n d_{k}}{r t t_{k}}\right)^{2}}, \frac{1}{c w n d_{i}}\right)
$$

As we mentioned in our previous paper, equation (2) can be derived by the assumption that the increase and decrease of congestion window sizes are balanced and that the virtual single TCP connections corresponding the subflows have the same throughput as the MPTCP connection [Kato et al., 2019].

\subsubsection{OLIA}

Khalili et al. mentioned two problems of LIA in their paper [Khalili et al., 2013]. The first problem is that LIA is not Pareto-optimal, i.e., the use of MPTCP may reduce the throughput of other TCPs without any benefit to the MPTCP throughput. The second problem is that MPTCP may be excessively aggressive toward TCP. In order resolve these problems, they proposed a new congestion control algorithm for MPTCP called the opportunistic linked-increases algorithm (OLIA). OLIA is specified in the following way.

OLIA defines $l_{i}$ for subflow $i$ as the larger among the number of transmitted bits over subflow $i$ between last two losses and the number of transmitted bits after the last loss. $r t t_{i}$ is defined as RTT on subflow $i$. It defines two set of subflows $M$ and $B$ as follows. 


$$
\begin{gathered}
M=\left\{k \mid k=\arg \max _{k} c w n d_{k}\right\} . \\
B=\left\{k \mid k=\arg \max _{k} \frac{l_{i}}{r t t_{k}{ }^{2}}\right\} .
\end{gathered}
$$

Here $M$ is the set of the subflows with largest congestion window, and $B$ is the set of the subflows that are presumably the best at the time.

Using those concepts, OLIA is defined in the following way.

- For each ACK segment over subflow $i$, increase $c w n d_{i}$ by

where $\alpha_{i}$ is calculated as follows:

$$
\frac{\frac{c w n d_{i}}{r t t_{i}{ }^{2}}}{\left(\sum_{k} \frac{c w n d_{k}}{r t t_{k}}\right)^{2}}+\frac{\alpha_{i}}{c w n d_{i}}
$$

$$
\alpha_{i}=\left\{\begin{array}{c}
\frac{1 / \text { number of subflows }}{|B \backslash M|} \text {, if } i \in B \backslash M \neq \emptyset \\
-\frac{1 / \text { number of subflows }}{|M|} \text {, if } i \in M \text { and } B \backslash M \neq \varnothing \\
0, \text { otherwise. }
\end{array}\right.
$$

$B \backslash M$ is the set of elements in $B$ but not in $M, \emptyset$ is the empty set, and $|B \backslash M|$ and $|M|$ are the number of elements of individual set.

- For each loss over subflow $i$, decrease $c w n d_{i}$ by $c w n d_{i} / 2$.

By comparing equations (3) and (6), we can say that LIA and OLIA are quite similar. Difference is that OLIA introduces the second term in equation (6), and that OLIA uses the value for the current subflow instead of the maximum as the numerator of the first term. Therefore, OLIA is still considered that it is based on TCP Reno similarly with LIA.

\subsubsection{BALIA}

Peng et al. proposed another congestion control algorithm focusing on a good balance between friendliness and responsiveness. This algorithm is called the balanced linked adaptation (BALIA), which is applied to the AIMD of the congestion avoidance phase [Peng et al., 2016]. It should be noted that the minimum value of ssthresh is set to one packet instead of 2 packets when more than one subflows are available.

Similarly with the above, let cwnd be the congestion window size of subflow $i$, and $r t t_{i}$ be the RTT of subflow $i$. The window adaptation of BALIA

- For each ACK segment over subflow $i$, increase $c w n d_{i}$ by:

$$
\frac{\frac{c w n d_{i}}{r t t_{i}{ }^{2}}}{\left(\sum_{k} \frac{c w n d_{k}}{r t t_{k}}\right)^{2}} \times \frac{1+\alpha_{i}}{2} \times \frac{4+\alpha_{i}}{5} .
$$

- For each loss over subflow $i$, decrease $c w n d_{i}$ by:

$$
\frac{c w n d_{i}}{2} \times \min \left(\alpha_{i}, 1.5\right) \text {. }
$$

Here, $\alpha_{i}=\frac{\max _{k} \frac{c w n d_{k}}{r t t_{k}}}{\frac{c w n d_{i}}{r t t_{i}}}$.

Similarly with OLIA, BALIA is also an extension of TCP Reno. Especially, when there is only one subflow, $\alpha_{i}$ is 1 and the increase and decrease of BALIA is identical to those of TCP Reno.

\section{EXPERIMENTAL SETTINGS}

Figure 3 shows the network configuration used in our experiment. An MPTCP data sender and a TCP (single path TCP) sender are connected to a 100 Mbps Ethernet hub. MPTCP sender has two Ethernet interfaces. A data receiver for MPTCP and TCP is connected to the hub through a bridge, which limits a data transmission rate to $25 \mathrm{Mbps}$. All nodes are running the Linux operating system, whose distribution is Ubuntu 16.04. 


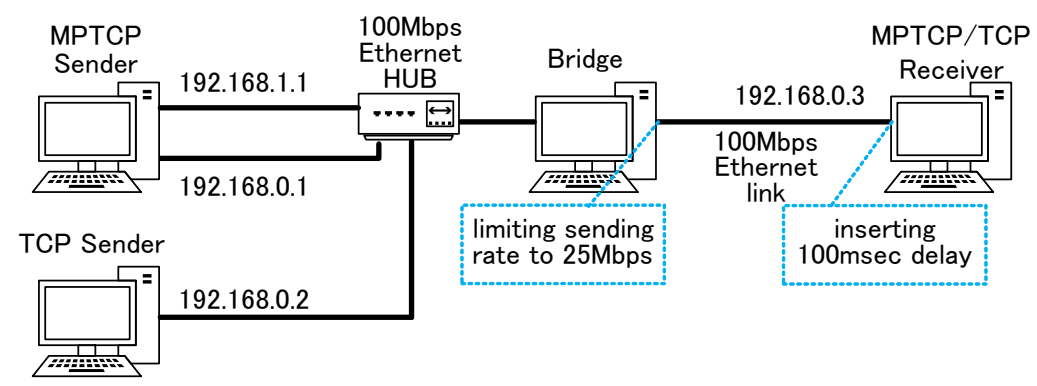

Figure 3. Network configuration used in experiment

Both MPTCP sender and receiver use an MPTCP software whose version is 0.94 stable, which is the newest version we can obtain [GitHub, 2019].

The IP addresses assigned to the network interfaces are shown in Figure 3. It should be noted that the LAN used in the experiment has subnet address 192.168.0.0/24, and the second Ethernet interface in the MPTCP sender another subnet 192.168.1.0/24. In the MPTCP sender side, the routing table needs to be specified for individual interfaces by using ip command. In the receiver side, a route entry to subnet 192.168.1.0/24 needs to be specified explicitly.

In the experiment, one MPTCP connection between the MPTCP sender and the receiver and one TCP connection between the TCP sender and the receiver are evaluated. In order to emulate a wide area Internet communication, $100 \mathrm{msec}$ delay is inserted at the receiver. The bridge limits the data transmission rate from the senders to the receiver into $25 \mathrm{Mbps}$. Here, an actual congestion will occur. As for the congestion control algorithm, the MPTCP sender uses LIA, OLIA, or BALIA, and the TCP sender uses TCP Reno or CUBIC TCP. In actual measurement runs, iperf is used for data transfer whose communication duration is ten second in one measurement run.

\section{RESULTS OF PERFORMANCE EVALUATION}

\subsection{Results of LIA for MPTCP Connection}

First, we conducted a performance evaluation with LIA for an MPTCP flow and TCP Reno for a single path TCP (SPTCP) flow. We executed ten measurement runs and obtained the average and standard deviation for MPTCP and SPTCP, which are 1.56 Mbps and 0,02 Mbps, and 21.6 Mbps and $0 \mathrm{Mbps}$. These results means that MPTCP with LIA is weaker than SPTCP with TCP Reno.

Figure 4 shows the details of communication in one measurement run. Figure 4(a) gives the sequence number vs. time for two MPTCP subflows and an SPTCP. As this figure indicates, the increase of the sequence number of two subflows are very slow. Figure 4(b) gives the congestion window size vs. time for two subflows and one TCP. In this case, the congestion window size of two subflows keeps 10 packets, which is the initial value of congestion window size.

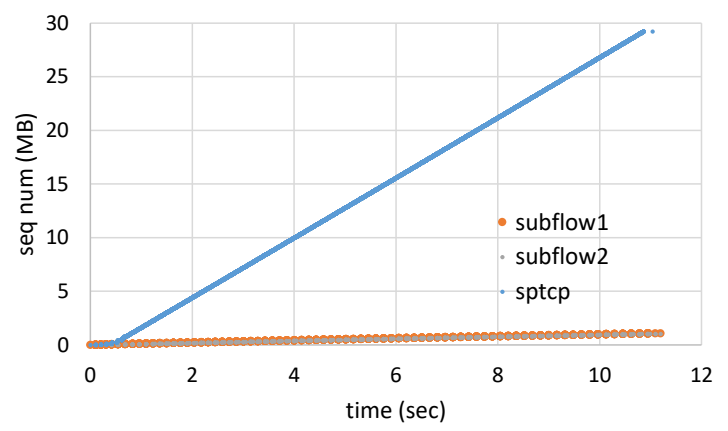

(a) Sequence number vs. time

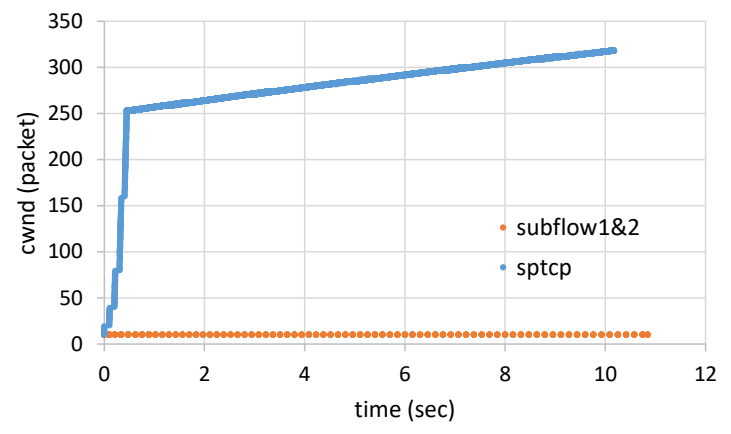

(b) Congestion window size vs. time

Figure 4. Result for LIA and TCP Reno 
We also conducted a performance evaluation with LIA for MPTCP and CUBIC TCP for SPTCP. Similarly we executed ten measurement runs. The average and standard deviation are $3.66 \mathrm{Mbps}$ and 2.82 Mbps for MPTCP, and 19.6 Mbps and 2.83 Mbps for SPTCP, respectively. In this case, MPTCP with LIA is also weaker than SPTCP with CUBIC TCP. The standard deviation is larger for LIA and CUBIC than for LIA and Reno.

Figures 5 and 6 show the details of one measurement run. Figure 5 gives the sequence number vs. time for two subflows and one SPTCP flow. Figure 5(a) shows that the sequence number of SPTCP increases rapidly compared with those of subflows. Figure 5(b) is a magnified graph for two subflows. The increase of two subflows is similar. Figure 6 give the congestion window size vs. time for two subflows and one SPTCP flow. From Figure 6(a), it is confirmed that the increase of congestion window size in SPTCP CUBIC TCP is very active, but on the other hand, those in two subflows are very slow. Figure 6(b) is a magnified graph for subflows. Three TCP connections suffer from packet losses around two second, when the congestion window size decreases. Before and after the packet losses, the increase of congestion window size is large for CUBIC TCP and low for LIA.

\subsection{Results of OLIA for MPTCP Connection}

Next evaluation is for OLIA and TCP Reno/CUBIC TCP. We conducted ten measurement runs. When OLIA and TCP Reno are used together, the throughput (average and standard deviation) is $15.8 \mathrm{Mbps} / 0.12 \mathrm{Mbps}$ for OLIA and 7.51 Mbps/0.17 Mbps for TCP Reno. That means OLIA provides higher throughput than TCP Reno. Figure 7 shows the details of one measurement run. Figure 7(a) is the sequence number vs. time and Figure $7(\mathrm{~b})$ is the congestion window size vs. time. The growth of sequence number and congestion window is large for one subflow, and small for the other subflow. The growth for the single path TCP is in between of them. As a result, the total throughput of MPTCP connection becomes higher than that of SPTCP connection.

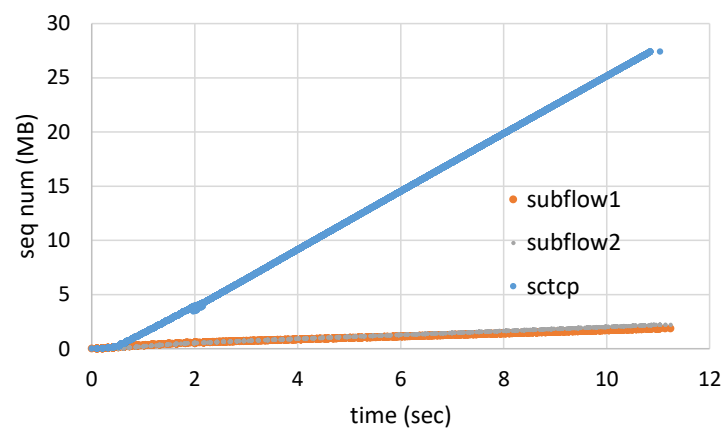

(a) for MPTCP and SPTCP

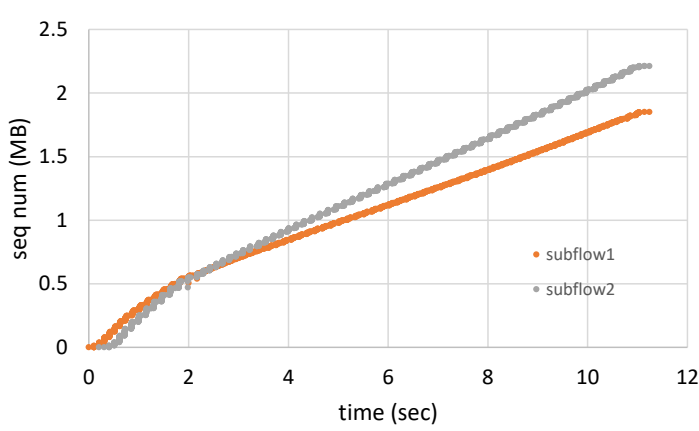

(b) for two MPTCP subflows

Figure 5. Result of sequence number vs. time for LIA and CUBIC TCP

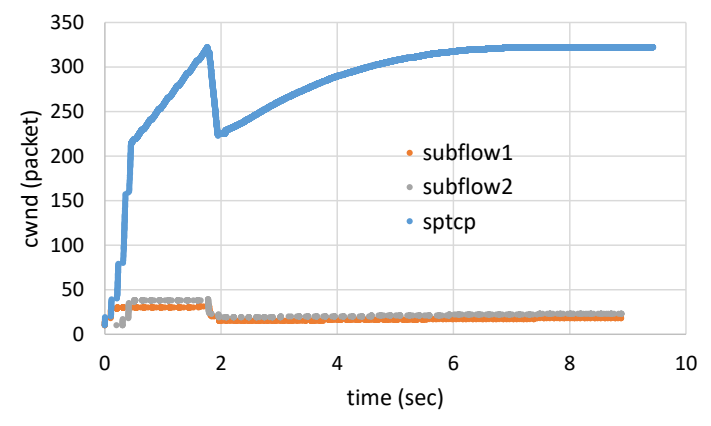

(a) for MPTCP and SPTCP

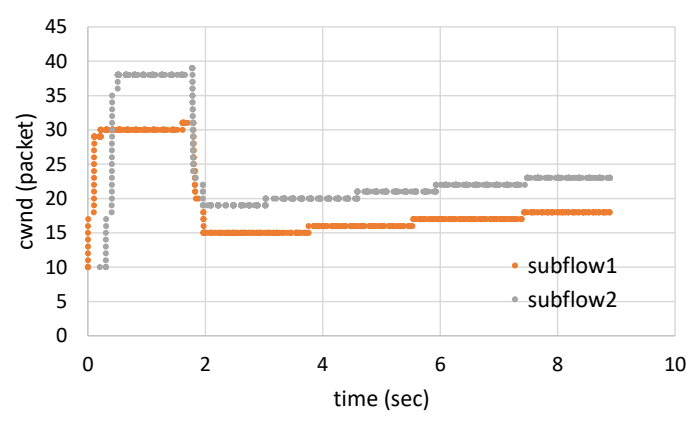

(b) for two MPTCP subflows

Figure 6. Result of congestion window size vs. time for LIA and CUBIC TCP 


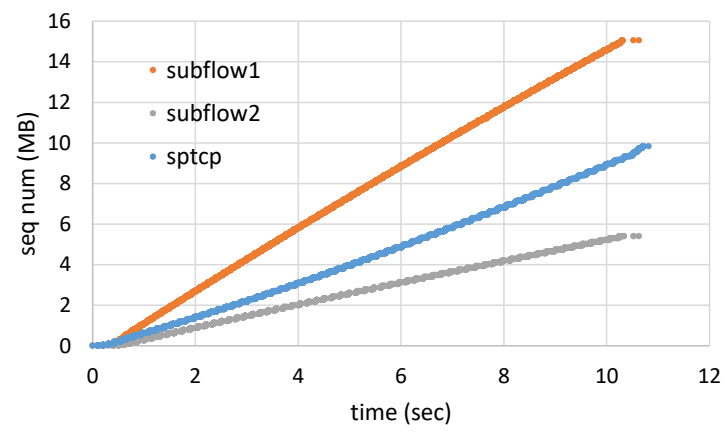

(a) Sequence number vs. time

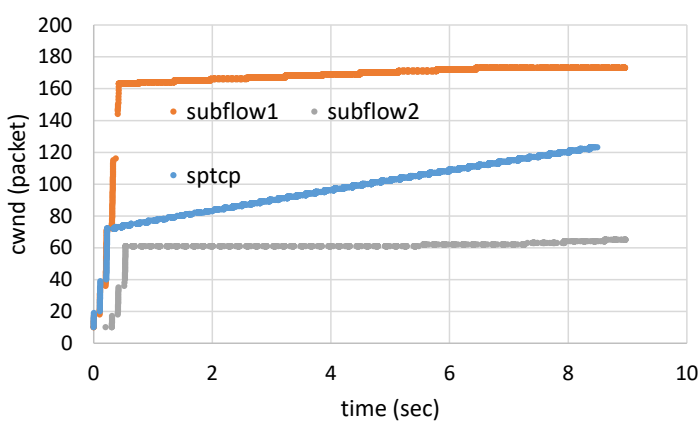

(b) Congestion window size vs. time

Figure 7. Result for OLIA and TCP Reno

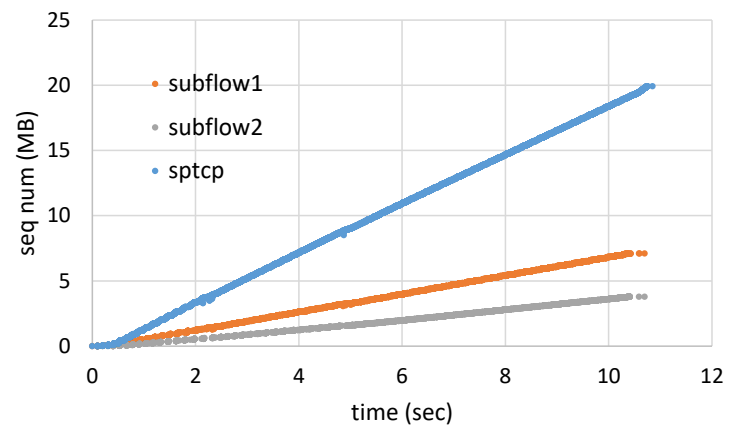

(a) Sequence number vs. time

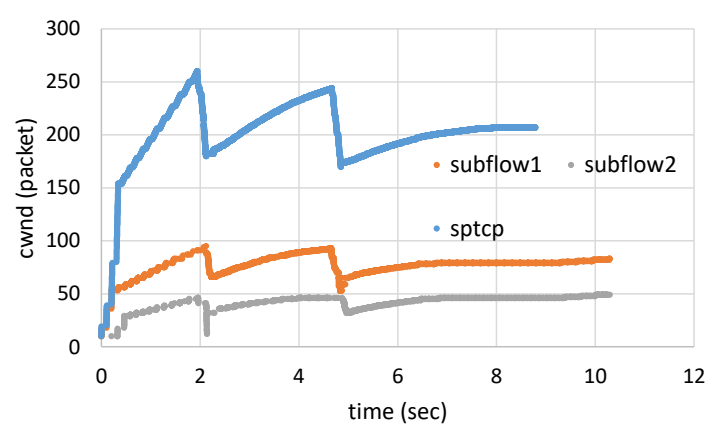

(b) Congestion window size vs. time

Figure 8. Result for OLIA and CUBIC TCP

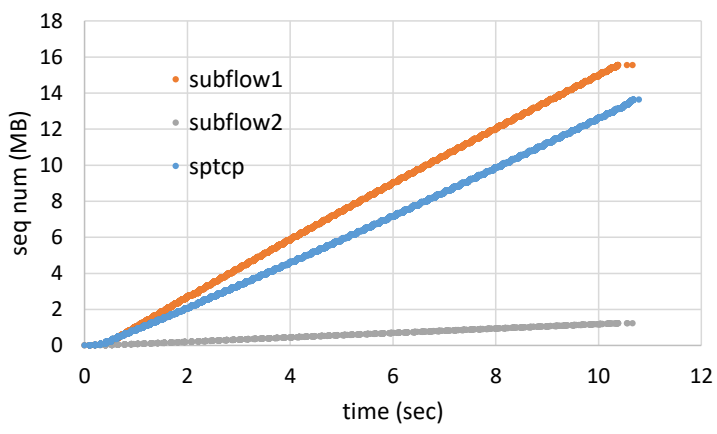

(a) Sequence number vs. time

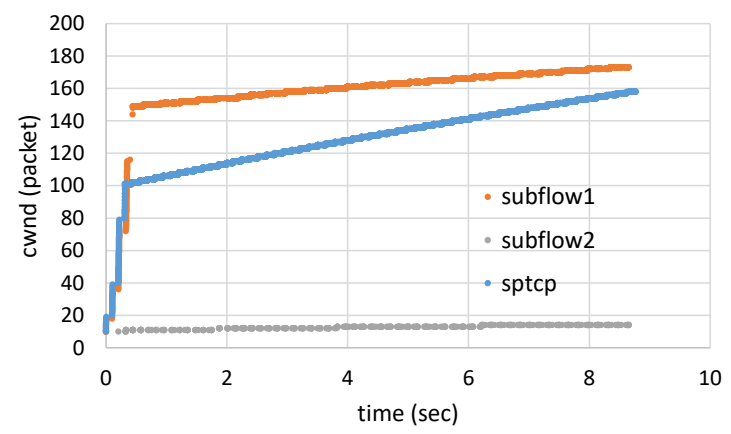

(b) Congestion window size vs. time

Figure 9. Result for BALIA and TCP Reno

When OLIA and CUBIC TCP are used together, the throughput (average and standard deviation) is 4.40 Mbps/2.83 Mbps and 18.8 Mbps/2.76 Mbps, respectively. In this case, SPTCP with CUBIC TCP provides higher throughput than OLIA MPTCP. Figure 8 shows the details of one measurement run. The growth of sequence number and congestion window size is larger than that of MPTCP subflows, and as a result the throughput is lower for OLIA than for CUBIC TCP.

\subsection{Results of BALIA for MPTCP Connection}

The last evaluation for BALIA and TCP Reno/CUBIC TCP. When BALIA and TCP Reno are used together, the throughput (average and standard deviation) is $12.9 \mathrm{Mbps} / 0.35 \mathrm{Mbps}$ for OLIA and $10.4 \mathrm{Mbps} / 0.27$ Mbps for TCP Reno. Similarly with OLIA, MPTCP provides higher throughput than Reno. Figure 9 shows 
the details of one measurement run, which indicates that both the sequence number and the congestion window size grow faster for BALIA, but the growth speed is similar in this case.

When BALIA and CUBIC TCP are used together, the throughput (average and standard deviation) is 4.43 Mbps/2.16 Mbps and 18.8 Mbps/2.16 Mbps, respectively. The result are very similar with the case of OLIA and CUBIC. The details of one measurement run shown in Figure 10 are similar with the case of OLIA.

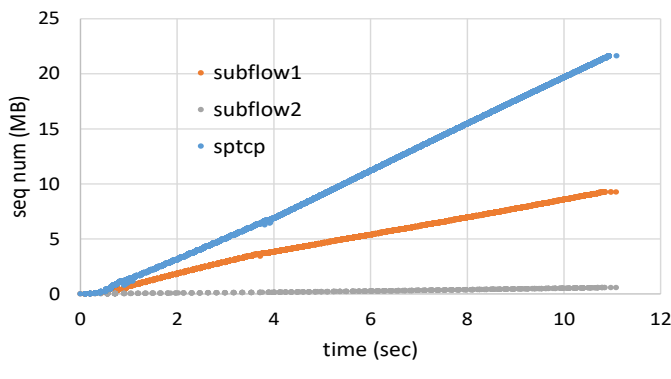

(a) Sequence number vs. time

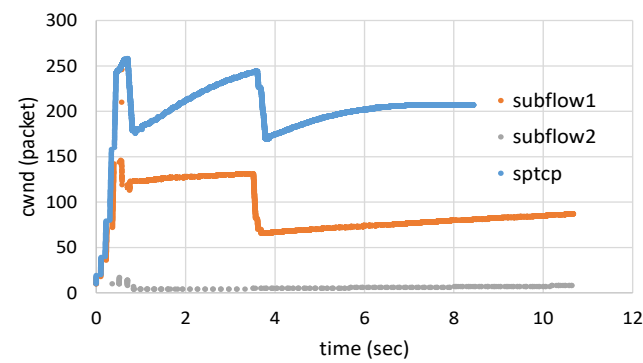

(b) Congestion window size vs. time

Figure 10. Result for BALIA and CUBIC TCP

\section{CONCLUSIONS}

In this paper, we presented the results of performance evaluation for MPTCP congestion control algorithms, LIA, OLIA, and BALIA in the condition that a single path TCP flow with TCP Reno or CUBIC TCP is used over a congested link. LIA, which is the standard congestion control algorithm of MPTCP, provides poor throughput when it coexists with either TCP Reno or CUBIC TCP. On the other hand, OLIA and BALIA provide similar or higher throughput than TCP Reno, but their throughput is lower than that of CUBIC TCP.

\section{REFERENCES}

AppleInsider Staff, 2019. Apple found to be using advanced Multipath TCP networking in iOS 7. http://appleinsider.com/articles/13/09/20/apple-found-to-be-using-advanced-multipath-tcp-networking-in-ios-7.

Floyd, S., Henderson, T., and Gurtov, A., 2004. The NewReno Modification to TCP's Fast Recovery Algorithm. IETF RFC 3728.

Ford, A., Raiciu, C., Handley, M., and Bonaventure, O., 2013. TCP Extensions for Multipath Operation with Multiple Addresses. IETF RFC 6824.

Ford, A., Raiciu, C., Handley, M., Barre, S., and J. Iyengar, J., 2011. Architectural Guidelines for Multipath TCP Development. IETF RFC 6182.

GitHub, 2019. Linux Kernel implementation of MultiPath TCP. https://multipath-tcp.org.

Ha, S., Rhee, I., and Xu, L., 2008. CUBIC: A New TCP-Friendly High-Speed TCP Variant. ACM SIGOPS Operating Systems Review, vol. 42, no. 5, pp. 64-74.

icteam, 2019. MultiPath TCP - Linux Kernel implementation, Users: Android. https://multipathtcp.org/pmwiki.php/Users/Android.

Kato, T., Diwakar, A., Yamamoto, R., Ohzahata, S., and Suzuki, N., 2019. Performance Evaluation of MaltiPath TCP Congestion Control. ICN 2019: 18th International Conference on Networks, pp. 19-24.

Khalili, R., Gast, N., Popovic, M., and Boudec, J., 2013. MPTCP Is Not Pareto-Optimal: Performance Issues and a Possible Solution. IEEE/ACM Trans. Networking, vol. 21, no. 5, pp. 1651-1665.

NGNM Alliance, 2015. NGMN 5G White Paper. https://www.ngmn.org/fileadmin/ngmn/content/images/news /ngmn_news/NGMN_5G_White_Paper_V1_0.pdf.

Paasch, C., and O. Bonaventure, O., 2014. Multipath TCP. Communications of the ACM, vol. 57, no. 4, pp. 51-57.

Peng, Q., Valid, A., Hwang, J., and Low, S., 2016. Multipath TCP: Analysis, Design and Implementation. IEEE/ACM Trans. Networking, vol. 24, no. 1, pp. 596-609.

Raiciu, C., Handley, M., and Wischik, D., 2011. Coupled Congestion Control for Multipath Transport Protocols. IETF RFC 6356.

Tan, K., Song, J., Zhang, Q., and Sridharan, M., 2006. A Compound TCP Approach for High-speed and Long Distance Networks. IEEE INFOCOM 2006, pp. 1-12. 